\title{
O PESO DO VAREJO, O PESO NO VAREJO E A IDENTIDADE: UMA ANÁLISE DE CONSUMIDORAS PLUS SIZE
}

\author{
The weight of retail, the weight in retail and identity: an analysis of plus size \\ consumers \\ El peso en el comercio, el peso en comercio y la identidad: un análisis de las \\ consumidoras plus size
}

\section{MARIA CAROLINA ZANETTE carolzanette@gmail.com Doutoranda em Administração de Empresas pela Escola de Administração de Empresas de São Paulo, Fundação Getulio Vargas - São Paulo - SP, Brasil}

\section{CARLOS EDUARDO LOURENÇO caerib@gmail.com Doutorando em Administração de Empresas pela Escola de Administração de Empresas de São Paulo, Fundação Getulio Vargas - São Paulo - SP, Brasil}

\section{ELIANE PEREIRA ZAMITH BRITO}

elianezbrito@gmail.com Professora da Escola de Administração de Empresas de São Paulo, Fundação Getulio Vargas São Paulo - SP, Brasil

\section{RESUMO}

Este trabalho estuda o conceito de identidade sob a ótica do consumo feminino de moda plus size. Foram realizadas 14 entrevistas fenomenológicas, observadas por uma perspectiva hermenêutica. Três categorias temáticas emergiram dos dados: a construção da identidade pela moda, o que envolve ser plus size e estratégias criativas para lidar com a configuração do portfólio de produtos no varejo, que não favorece mulheres plus size. Entre os principais resultados, destacam-se a forma pela qual o termo plus size age como estigma, influenciando os projetos identitários das consumidoras, o papel do varejo na estigmatização e a saga quase epopeica das compras, que envolve até um "mercado negro" com as vendedoras.

PALAVRAS-CHAVE | Identidade, moda, cultura de consumo, varejo de moda, estigma.

\section{ABSTRACT}

This paper examines the concept of identity from the perspective of plus size women's consumption of fashion. 14 phenomenological interviews were conducted and analyzed from a hermeneutical perspective. Three thematic categories emerged from the data analysis: the construction of identity through fashion, elements of plus size identity and creative strategies to deal with the lack of products for plus size women in retail. Among the main results, the way the term plus size acts as stigma, influencing consumer's identity projects, the role of retail in the stigmatization process and the shopping epic saga, which involves a "black market" with the participation of sellers.

KEYWORDS I Identity, fashion, consumer culture, fashion retail, stigma.

\section{RESUMEN}

Este trabajo estudia el concepto de identidad bajo la óptica del consumo femenino de moda plus size. Se realizaron 14 entrevistas fenomenológicas, observadas por una perspectiva hermenéutica. Tres categorías temáticas emergieron de los datos: la construcción de la identidad por la moda, lo que envuelve ser plus size y las estrategias creativas para lidiar con la configuración del portafolio de productos en el comercio minorista, que no favorece a las mujeres plus size. Entre los principales resultados, se destacan la forma por la cual el término plus size actúa como estigma, influenciando los proyectos identificadores de las consumidoras, el papel del mercado minorista en la estigmatización y la saga casi epopeyita de las compras, que envuelve hasta un "mercado negro" con las vendedoras.

PALABRAS-CLAVE / Identidad, moda, cultura de consumo, comercio minorista de moda, estigma. 


\section{INTRODUÇÃO}

O conceito de identidade é um dos temas centrais na área de comportamento do consumidor (ARNOULD e THOMPSON, 2005). Uma das abordagens adotadas para explicar esse conceito é aquela que relaciona as marcas e os produtos à identidade dos consumidores (GAO, WHEELER, SHIV, 2009; KLEINE, KLEINE, KERNAN, 1993; SCHAU, GILLY, WOLFINBARGER, 2009). Essa relação deve-se à teoria de que objetos são transmissores de discursos e ideologias presentes na cultura popular (DOUGLAS e ISHERWOOD, 1979; FIRAT e VENKATESH, 1995) e são utilizados pelos consumidores para construir suas identidades (BELK, 1988).

Além de envolver objetos e marcas, o varejo também é um elemento influenciador do processo constitutivo da identidade dos consumidores. Tal argumento baseia-se em trabalhos recentes que indicam que o varejo é um transmissor de ideologias (BORGHINI e outros, 2009) ou discursos culturais (THOMP. SON, RINDFLEISCH, ARSEL, 2006), com os quais o consumidor, criativamente, constrói seu senso de "eu" (FIRAT e VENKATESH, 1995). Contudo, outros estudos mostram que existem consumidores estigmatizados e preteridos pelo mercado (SANDICKI e GER, 2013; SCARABOTO e FISCHER, 2013), que, ainda que desejem adquirir determinado produto, e mesmo que possam pagar por ele, não o encontram no varejo.

0 presente trabalho examina a relação entre a identidade, o estigma e o papel do varejo nessa relação a partir da ótica de consumidoras plus size, ou seja, mulheres que utilizam roupas com numeração igual ou superior a 44 (WINN, 2004) e que se consideram acima do peso tido como "ideal". Especificamente, examinamos como o estigma do peso afeta a criação de um projeto identitário (SHANKAR, ELLIOT, FITCHETT, 2009) constituído por meio do eu-estendido pelas posses (BELK, 1988) e quais estratégias as consumidoras criam para levar a cabo esse projeto diante de um dos principais elementos de mercado reprodutores do discurso de estigmatização, que é o varejo.

0 artigo contribui com a literatura de cultura de consumo, conjugada majoritariamente a técnicas interpretativistas (GAIÃO, SOUZA, LEÃO, 2012), ainda pouco exploradas no Brasil (SAMPAIO e outros, 2012).

Este trabalho está dividido em seis partes. Na primeira, apresentamos conceitos relacionados à identidade no consumo e ao varejo. Na segunda seção, discutimos os métodos que adotamos para a realização do trabalho e, na seguinte, apresentamos os resultados da pesquisa empírica. A quarta seção contém a discussão teórica dos achados e a relação com a área de varejo, e, por fim, apresentamos as limitações e sugestões para pesquisas futuras.

\section{BASE TEÓRICA}

Esta seção está organizada em duas partes: a primeira trata identidade e consumo de moda e a segunda parte refere-se ao papel do varejo como meio para transmitir significado.

\section{Identidade, corpo e (não) consumo}

A identidade, na contemporaneidade, é um projeto dos indivíduos, que se desenvolve continuamente por meio de um determinado processo (SHANKAR, ELLIOT, FITCHETT, 2009). O projeto identitário envolve a tentativa de criação de um self coerente, ainda que fragmentado (FIRAT e VENKATESH, 1995) e diversificado (BAHL, 2013).

A cultura de consumo é uma arena para a produção e circulação de representações identitárias (SHANKAR, ELLIOT, FITCHETT, 2009), sendo o mercado a fonte de recursos simbólicos pelos quais as pessoas constroem narrativas de identidade (ARNOULD e THOMPSON, 2005; SCHAU e GILLY, 2003), expressando soberania (HOLT, 2002), individualidade (THOMPSON e HAYTKO, 1997) e características que as distinguem de outros grupos (ARSEL e THOMPSON, 2011; KATES, 2002; SCHOUTEN e MCALEXANDER, 1995).

Belk, em 1988, cunhou a expressão "self estendido", em um trabalho seminal descrevendo formas pelas quais posses ou símbolos, como lugares, tornam-se parte do que um indivíduo é. É válido notar que, em Belk (1988), self, senso de self e identidade são tratados como sinônimos e indicam a maneira pela qual o indivíduo percebe-se a si mesmo. Posteriormente, foi investigado como consumidores utilizam objetos (KATES, 2002), práticas de consumo (KOZINETS, 2002), ícones culturais (KOZINETS, 2001) e marcas (FOURNIER, 1998; LUEDICKE, THOMPSON, GIESLER, 2009) em seus projetos identitários.

A extensão do self por meio de objetos de consumo em busca de um projeto identitário acontece em um contexto de interação entre indivíduos e grupos de indivíduos. 0 consumidor que investe em determinado projeto está sujeito ao julgamento alheio, de modo que alteridade (OLIVEIRA e LEÃO, 2012) tem um papel fundamental na construção e na legitimação (SANDICKI e GER, 2010) do projeto identitário levado a cabo pelo consumidor.

Se da interação social, embutida em discursos ideológicos, emergem avaliações negativas e estereotipadas de determinado grupo de consumidores, esses serão considerados estigmatizados (SANDICKI e GER, 2013) e, provavelmente, afastados de certos ambientes de convívio (GOFFMAN, 1963). Estudos que consideram a identidade de consumidores estigmatizados por características físicas (SCARABOTO e FISCHER, 2013), 
orientação sexual (KATES, 2002) ou escolhas de consumo (KOZINETS, 2001; SANDICKI e GER, 2010) têm se concentrado nas tensões, provocadas por posses, que levam o indivíduo a se identificar mais com o grupo estigmatizado ou a se distanciar de seus estereótipos.

Conforme indicam Scaraboto e Fischer (2013), os consumidores plus size podem ser considerados um grupo estigmatizado. Além disso, são estigmatizados por uma característica considerada controlável, que é o próprio corpo ou partes dele. 0 corpo é parte do self estendido (BELK, 1988), podendo ser modificado (ALMEIDA, 2006) ou controlado (THOMPSON e TROESTER, 2002). O corpo é o palco onde significados de marcas e objetos manifestam-se (FONTENELLE, 2008) e é objeto de discursos simbólicos de juventude e beleza (THOMPSON e HIRSCHMAN, 1995). Por ser considerado controlável, o indivíduo cujo corpo colide com símbolos esperados tende a ser estigmatizado, inclusive moralmente, sendo o culpado pela lassidão moral com que trata uma das principais posses de seu self.

\section{Varejo como transmissor de significados e seu papel na formação identitária do consumidor}

Uma das principais manifestações do processo de transmissão de discursos por meio do mercado são as lojas do varejo, protagonistas da vida contemporânea, por serem espaços, ao mesmo tempo, de lazer e consumo (ALBINO e outros, 2010).

Estudos contemporâneos na área de cultura de consumo indicam que o varejo, a exemplo dos bens, transmite significados e mesmo discursos ideológicos (BORGHINI e outros, 2009; DION e ARNOULD, 2011; THOMPSON, RINDFLEISCH, ARSEL, 2006). Esses discursos são transmitidos por meio da atmosfera do varejo, da disposição dos objetos na área de venda, das cores, do mix de produtos, entre outros elementos (BORGHINI e outros, 2009) relevantes na decisão da escolha da loja pelo consumidor (HAYTKO e BAKER, 2004).

A transmissão desses significados pelo varejo dá-se tanto por sua comunicação, que pode contribuir com a legitimação da marca (ARNOLD, KOZINETS, HANDELMAN, 2001), quanto pela experiência do consumidor nesse ambiente (BORGHINI e outros, 2009; DION e ARNOULD, 2011). O conceito de experiência é a resposta subjetiva de qualquer consumidor para qualquer contato com uma companhia, seja ele direto - compra ou uso de serviços - ou indireto - encontros não planejados, incluindo propaganda, comentários, entre outros (MEYER e SCHWAGER, 2007). Assim, não só o varejo transmitirá significados, como obterá respostas dos consumidores com base em suas experiências, interpretadas e elaboradas também conforme os projetos identitários individuais.
Como o eu-estendido não depende apenas de objetos físicos e tangíveis para ser consolidado (BELK, 1988), ainda que a posse de tais objetos crie relações importantes para ele (AHUVIA, 2005), a associação da identidade com uma loja pode acontecer. Contudo, se há escassez de produtos e os consumidores não são atendidos pelo varejo, como é o caso de consumidores plus size (SCARABOTTO e FISCHER, 2013), a posse não se consolida, e o consumidor pode enfrentar tensões identitárias, como no caso das mulheres turcas, que buscavam alternativas fashion para uma moda que contemplasse o uso do véu.

Nesse contexto, o varejo focado em plus size pode potencializar a propensão de o consumidor assumir a condição de estigmatizado (GOFFMAN, 1963), segregando-o como consumidor de moda. Por outro lado, tal ambiente de varejo pode servir como um mecanismo de proteção para o estigmatizado: primeiro, inserindo o consumidor de moda plus size em um contexto de pares, mitigando a condição de estigmatizado; segundo, como salienta Goffman (1963, p. 144), os não estigmatizados podem oferecer a cortesia de não dar importância ao seu “defeito", levando-o a uma percepção de inclusão e a sentir-se aceito como os outros.

\section{PROCEDIMENTOS METODOLÓGICOS}

Esta seção está dividida em três partes: na primeira, discutiremos as considerações epistemológicas da pesquisa, que norteiam a coleta e análise dos dados. Depois, descreveremos os procedimentos de coleta. Por fim, explicaremos os procedimentos de análise.

\section{Considerações epistemológicas}

A execução do trabalho empírico foi realizada sob a premissa de que a identidade e os significados de consumo relacionamse dialeticamente (THOMPSON, 1997), como parte da experiência do consumidor (THOMPSON, LOCANDER, POLLIO, 1989), dentro de um contexto que a situa (ASKEGAARD e LINNET, 2011). Sob tal premissa, buscamos narrativas que evidenciassem "significados imbricados nas práticas comuns da vida cotidiana” (SANTOS e PINTO, 2008, p. 9) dos consumidores.

Thompson, Locander e Pollio (1989) argumentam que a fenomenologia existencial é uma forma de trazer a experiência à pesquisa do consumidor e desenvolvem um método de entrevistas fenomenológicas amplamente utilizado em trabalhos posteriores (THOMPSON e HAYTKO, 1997; THOMPSON e TROESTER, 2002). Já Thompson (1997) desenvolve um esquema hermenêutico considerado adequado pelo autor para a análise de tais entrevistas. 
Nossa abordagem é similar à de Thompson (1997) e de outros trabalhos que indicam o uso de um círculo hermenêutico (ARNOLD e FISCHER, 1994; MOISANDER e VALTONEN, 2006; THOMPSON, LOCANDER, POLLIO, 1989), ou seja, de análises profundas e concomitantes do todo e das partes das narrativas dos consumidores, buscando hermeneuticamente a interpretação dos sentidos (BLEICHER, 1980). Os autores fundem horizontes com o texto e envolvem-se ativamente na tarefa de compreender holisticamente as histórias contadas pelos consumidores (ARNOLD e FISCHER, 1994; THOMPSON, 1997).

\section{Método de coleta}

Para acessar os dados, realizamos entrevistas fenomenológicas em profundidade, formatadas como um diálogo, sem roteiro pré -estruturado e evitando questões que usem "por quê", de modo a incentivar a entrevistada a desenvolver suas ideias e evitar racionalizações sobre suas experiências de vida (THOMPSON, LOCANDER, POLLIO, 1989). As entrevistas geraram narrativas (HOPKINSON e HOG, 2006) das histórias de vida das consumidoras e sua relação com a moda.

Foram entrevistadas 14 mulheres, residentes em diferentes cidades do estado de São Paulo. Os perfis das entrevistadas encontram-se descritos no Quadro 1. A decisão de entrevistar mulheres relaciona-se com a ligação entre a moda, a beleza e a feminilidade na sociedade contemporânea (THOMPSON e HAYTKO, 1997). Duas das entrevistas foram conduzidas com mais de uma pessoa ao mesmo tempo: a de Laura e Otávia, que são irmãs, e a de Regina e Francine, mãe e filha. Tal procedimento trouxe a vantagem de relatos de experiências compartilhadas e situações vividas conjuntamente. Contudo, no caso da entrevista de Regina e Francine, a figura de autoridade da mãe pode ter inibido a filha quanto a revelar certas experiências. Buscamos mulheres que aparentavam estar acima do peso considerado "adequado". Não selecionamos o que é ser plus size necessariamente pelo número do manequim, já que não há um consenso sobre o tema, ainda que boa parte das referências indique que o manequim mínimo plus size seja 44 (WINN, 2004). Contudo, ao explicitar para as entrevistadas o propósito do trabalho, deixamos claro o tema sobre o qual seria a entrevista, de modo que nosso prejulgamento sobre sua aparência foi revelado. As que rejeitaram explicitamente o rótulo de plus size ou, em nossa percepção, ocultaram o número de seus manequins (dois casos) não foram convidadas para o trabalho. Os nomes foram modificados para preservar suas identidades.
Quadro 1. Descrição das entrevistadas

\begin{tabular}{l|l|c|l}
\hline Nome & Profissão & Idade & Cidade \\
\hline Larissa & Professora universitária & 32 & São Paulo \\
\hline Otávia & Advogada & 27 & Ribeirão Preto \\
\hline Laura & Advogada & 26 & Ribeirão Preto \\
\hline Tarsila & Microempresária & 60 & Ribeirão Preto \\
\hline Regina & Empresária & 43 & Batatais \\
\hline Francine & Estudante - pré-vestibular & 17 & Batatais \\
\hline Tamires & Administradora de empresas & 32 & São Paulo \\
\hline Marina & Analista de marketing & 26 & São Paulo \\
\hline Kátia & Recepcionista & 18 & Jundiaí \\
\hline Patrícia & Analista financeira & 42 & Campinas \\
\hline Fabíola & Estudante de economia & 27 & Ribeirão Preto \\
\hline Catarina & Esteticista & 43 & Ribeirão Preto \\
\hline Marília & Professora de biologia & 46 & Ribeirão Preto \\
\hline Marlene & Professora de português & 59 & Ribeirão Preto \\
\hline
\end{tabular}

Realizamos entrevistas até que a variabilidade de informação não mais ocorresse e as entrevistadas reforçassem ideias já capturadas. As entrevistas foram gravadas e transcritas integralmente, totalizando mais de 10 horas de áudio e 88.738 palavras.

\section{Método de análise}

A análise foi realizada transitando entre o todo e as partes, visando ao entendimento da relação entre identidade e significados simbólicos transmitidos por objetos e lugares (varejo). Para tanto, todas as entrevistas foram transcritas verbatim e analisadas por dois dos três autores, dando-se maior atenção à análise do movimento narrativo, ou seja, à interação entre passado e presente nas entrevistas das consumidoras e nas influências culturais que pareceram afetar os seus projetos identitários (THOMPSON, 1997).

Após considerar cada entrevista como um todo, com seus próprios significados, dois dos autores, isoladamente, estabeleceram padrões, sequencialmente discutidos em grupo por todos os autores do estudo. Foi necessário voltar às partes (entrevistas) para elucidar certos aspectos da interpretação ou rever trechos que transmitiam significados relevantes para a análise como um todo. 0 processo repetiu-se até que uma interpretação integrada dos conceitos surgisse. 


\section{IDENTIDADE: MODA, PESO E O PAPEL DO VAREJO}

A análise das entrevistas resultou em três categorias temáticas: a construção da identidade pela moda, o que envolve ser plus size e estratégias criativas para lidar com a falta de produtos destinados a mulheres plus size no varejo. Tais categorias temáticas não são interpretadas isoladamente, mas se entrelaçam, formando um esquema conceitual representante dos discursos identitários das entrevistadas. 0 esquema conceitual é ilustrado na Figura 1.

Figura 1. Esquema conceitual: integração das categorias de análise

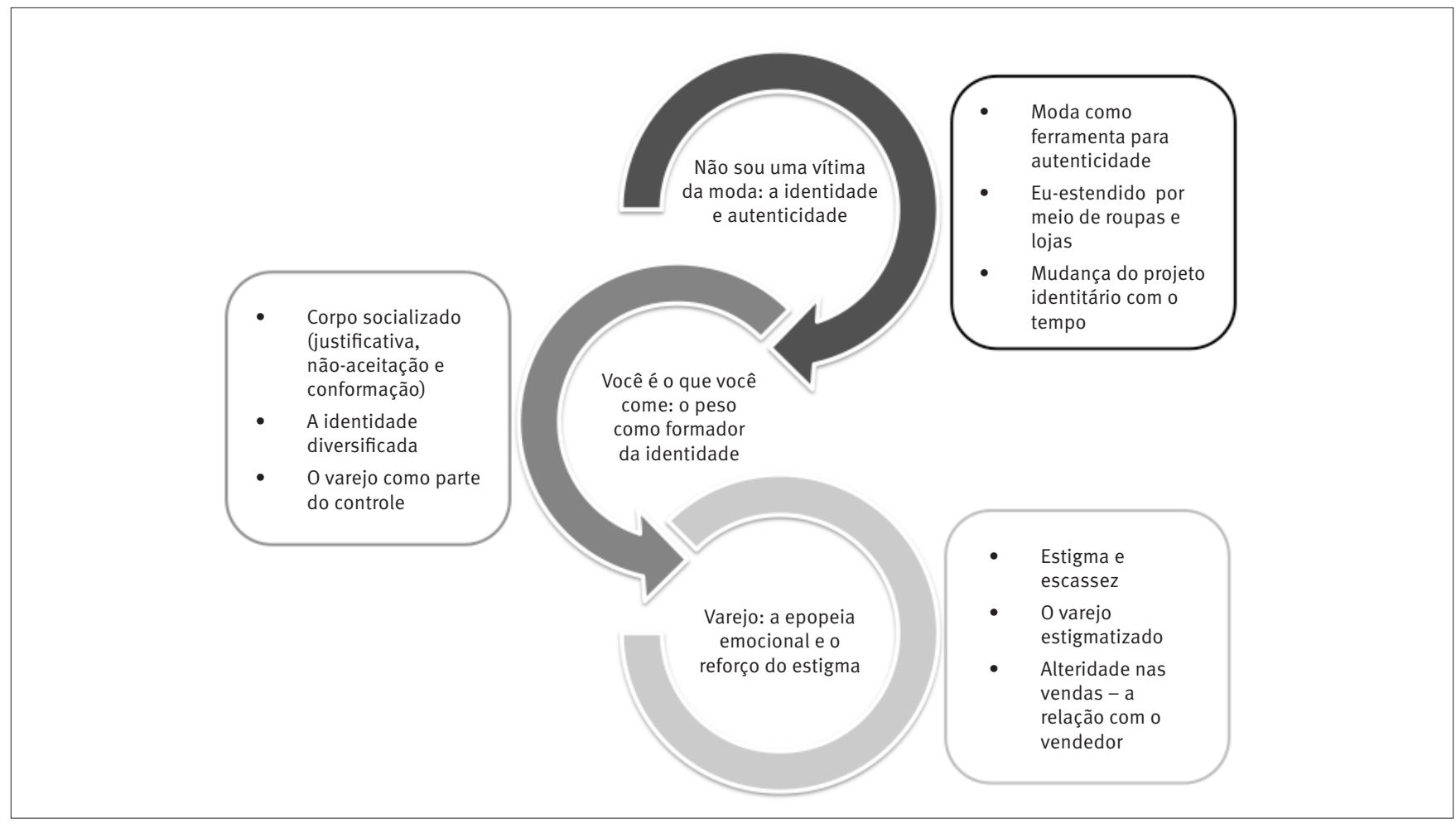

\section{Não sou uma vítima da moda: identidade e autenticidade}

A moda é um elemento central na identidade das consumidoras entrevistadas, sendo relevante para negociar o nível de autenticidade e de inclusão em relação a diferentes grupos sociais (THOMPSON e HAYTKO, 1997) e para apresentar-se ao mundo como uma pessoa mais magra. As ferramentas usadas para tanto são as peças de roupas, as marcas e as lojas frequentadas pelas consumidoras, que auxiliam a formação de seu eu-estendido.

No trecho abaixo, Marília fala de sua relação com a moda.

Já tive a fase da moda, modinha, que é o que estava sendo usado no momento, e aí eu queria que coubesse em mim de qualquer jeito. Hoje eu já sei que não dá pra ser assim, porque não é toda roupa que cai bem, então hoje, pra mim, moda é a roupa com que eu me sinto confortável, é claro que dentro de algumas coisas que estão sendo usadas, né?

Então, às vezes, calha de serem coisas que eu gosto muito, que nem, por exemplo, agora a boca mais larga, a calça mais reta... Mas hoje é aquilo que eu me sinto bem. Então é uma calça jeans, um camisetão, é uma bata...

0 trecho citado é representativo das narrativas da maioria das entrevistadas e ilustra dois aspectos da identidade de moda. Há a ideia de um estilo próprio e autêntico, que, como em Thompson e Haytko (1997), existe por estar em confronto com o que a entrevistada considera massificado ou "modinha", termo que significa a moda atual, do momento, vendida em todas as lojas, especialmente as de departamentos, mas que é percebida como pouco acessível ao público plus size, por não estar disponível em seu tamanho. 
O elemento contrário à “modinha” é o "estilo próprio”. 0 estilo próprio é o cerne da relação entre as entrevistadas e suas peças de roupa. 0 estilo, que liga as entrevistadas a marcas com as quais elas se relacionam (FOURNIER, 1998) e a objetos que transmitam significados (DOUGLAS e ISHERWOOD, 1979), varia conforme os projetos identitários de cada uma. 0 trecho de Larissa, que define seu estilo como "suburbana deslumbrada”, ilustra seu projeto identitário de ligação com a moda (no caso, com a moda em maquiagem) como uma refração ao que é considerado elitista, na tentativa de estabelecer um "eu" coerente (ARNOULD e THOMPSON, 2005). O estilo, conforme Murray (2002) indica, é um local onde políticas de diferença são marcadas.

O estilo suburbana deslumbrada é assim... eu hoje gosto muito de ver blog de moda, né? Eu vejo muitos blogs gringos... e blog de maquiagem eu acompanho muitos aqui, né? Mas blog de maquiagem classe $C$, não blog de maquiagem Dior, não, não é assim... as marcas Avon, Natura, Boticário, MAC... mas o máximo que chega é MAC, entendeu? Não vem Dior, Chanel, essas coisas não.

O trecho de Larissa também exemplifica o seu processo de aprendizado de moda. Esse "estilo pessoal" surge do aprendizado que ocorre durante a vida da entrevistada, o contexto no qual ela cresceu, as pessoas de seu relacionamento e o acesso a informações especializadas de moda. 0 aprendizado inclui o protocolo de vestimenta adequado a cada ocasião, as lojas onde comprar, as diversas possibilidades de combinações e as marcas escolhidas, condizentes com seu "estilo próprio" e, também, com o gerenciamento de impressões buscado por cada consumidora, de modo que a diferencie e, ao mesmo tempo, seja útil para minimizar tensões culturais e de classe.

$O$ aprendizado vem com a idade. $E$, também com a idade, vem uma mudança no que é considerado estilo próprio. As narrativas de moda ilustram projetos relacionados ao corpo das entrevistadas (MURRAY, 2002). Conforme as entrevistadas vão amadurecendo, os projetos de corpo mudam e o desejo de conformidade diminui, abrindo mais espaço para a consolidação de um estilo consistente e para a aceitação do próprio corpo. Além disso, a questão financeira passa a ser preponderante com o amadurecimento. 0 trecho abaixo, da entrevista de Marília, ilustra tais situações.

E ainda não foi só a adolescência, eu acho que a gente passa outro período de aceitação... até que chega um momento que você passa a se aceitar do jeito que você é... e valorizar outros aspectos, então você vai valorizando outros aspectos da roupa... por isso que eu acho que... em alguns momentos, é bom a gente ter condições de comprar a roupa quando a gente encontra... não ter que ficar usando só modinha, a gente pode criar um estilo pessoal, porque roupa é algo que você precisa se sentir bem dentro dela...

Como abordado, a identidade de moda não se separa da forma do corpo. Na próxima subseção, exploramos mais profundamente a relação das entrevistadas com seu peso.

\section{Você é o que você come: o peso como formador da identidade}

O peso é tido, geralmente, como uma fonte de angústia para as entrevistadas. Como em Thompson e Hirschman (1995), seus corpos são socializados, sendo sua imagem caracterizada por percepções condicionadas a relações sociais, prescrições normativas e significados morais, que julgam o corpo como algo que resulta da disciplina do espírito (THOMPSON e HIRSCHMAN, 1995; THOMPSON e TROESTER, 2002).

Esse processo de socialização do corpo envolve especialmente três dimensões: a justificativa, a não aceitação e a conformação do corpo com o que é considerado um corpo ideal. 0 comentário destacado de Francine é o exemplo de um discurso de justificativa. Francine aceita e entende o discurso que liga a magreza à beleza. Pela sua entrevista e pelos comentários de sua mãe, Regina, ser gorda já é parte de sua identidade: ela se assume como tal e parece aceitar a situação. Entretanto, ela sente a necessidade de se desvincular de um “espírito de gordo". Se, como Thompson e Hirschman (1995) afirmam, a gordura, na cultura popular, está associada à falta de cuidado com o próprio corpo - uma questão moral, que liga o cuidado ao corpo com a pureza do espírito - Francine busca justificar sua condição pelo seu estilo de vida (estudante de pré-vestibular), ao desvincular seu corpo da disciplina de seu espírito. Em suas palavras:

Eu comeria [...] só uma salada, (mas) eu tenho que reentrar na aula, sendo que eu saio 12 h3o. 13h3o eu tenho aula, não dá pra você sentar no restaurante e comer. Você come, sim, qualquer coisa. São calorias, né? Então, já tá engordando mais cedo pelo estilo de vida, e aí vai ficando mais difícil manter o peso. 
Justificar a condição de gorda parece uma estratégia para se desvincular da não aceitação do próprio corpo.

A relação de alteridade (OLIVEIRA e LEÃO, 2012) também é importante. As entrevistadas percebem que a reação da sociedade em geral em relação à gordura tende a ser negativa, Laura, por exemplo, mostra-se ofendida e sente-se estigmatizada (GOFFMAN, 1963).

Eu acho que a nossa sociedade é muito estética, realmente, é muito, eu vejo muita piadinha, fazem muitas piadinhas com o pessoal, com gordinhos aqui, e com gordinhas. Aliás, já teve amigos meus próximos que já fizeram assim: “Pô, mas você não perdoa nem a gorda?” E sai, assim, naturalmente. [...] As pessoas pensam assim, a maior parte delas. Eu não quero conviver com esse tipo de gente.

A não aceitação leva a estratégias de "defesa” contra o estigma da gordura, reforçando a ideia de uma identidade diversificada, da separação entre o espírito (inteligência) e o corpo (THOMPSON e HIRSCHMAN, 1995), como a supervalorização de outras faces da identidade pessoal, tais quais o sucesso acadêmico e profissional. 0 comentário de Larissa ilustra essa situação: “(Eu estava) bem grande mesmo [...] e assim, mas não me importava, não me achava feia nem nada, simplesmente não ligava para isso, eu achava que era coisa de quem não tinha o QI alto".

A não aceitação também leva a tentativas de emagrecer: entre as citadas, encontram-se o uso de anfetaminas para, pelo menos, cinco das entrevistadas, planos de implantar um balão gástrico, além de incontáveis dietas e exercícios físicos. É interessante notar que essas tentativas de emagrecer acontecem motivadas, também, pela possibilidade de escolher as roupas desejadas, da "modinha”, o que adiciona à tensão entre autenticidade e conformação (THOMPSON e HAYTKO, 1997) um novo elemento.

Com o amadurecimento, parece haver uma situação de conformação com a condição de plus size, ainda que esta não elimine as tensões de uma identidade fragmentada em busca de um todo coerente (ARNOULD e THOMPSON, 2005). Essa é uma situação de conformação com a condição de plus size. Não que o conflito com o corpo e a sensação de não ser um sujeito adequado à moda dissipem-se, no entanto essas consumidoras buscam legitimação para sua própria imagem e, muitas vezes, passam a ser ativas em propagar um novo discurso de padrão de beleza. 0 acesso a blogs especializados em moda vem se mostrando um elemento importante para es- sas consumidoras (SCARABOTO e FISCHER, 2013), facilitando o acesso a informações cujo aprendizado seria dificultado sem essas ferramentas on-line. 0 comentário de Otávia é um exemplo desse processo.

Eu sempre fui efeito sanfona, mais aí é um problema hormonal [...] Eu parei de comprar roupas, aí você fica com aquelas roupas ridículas, roupas que te espremem, que não são confortáveis. Aí você começa a parar de sair. Eu, pelo menos, começo a parar de sair. Aí em blog eu comecei aceitar e falar: “Não, bem, eu posso sair linda" e, realmente, foi melhorando minha autoestima. Aí eu voltei a comprar, eu parei de me privar por causa do peso e parei totalmente, porque eu estava me privando, sim.

O papel do varejo na não aceitação e conformidade é proeminente. Por um lado, os comentários das entrevistadas mostram descontentamento com a falta de ofertas (como o trecho de Fabíola, que encerra a subseção), o que as leva, entre outras razões já mencionadas, como o controle social do corpo, a tentativas de emagrecer. Por outro lado, a oferta de lojas plus size traz sentimentos ambíguos, pois força-as a encarar seus projetos identitários como consumidoras que se dizem gordas (conformação), o que leva a dilemas morais relacionados com o fracasso do emagrecimento. A relação das entrevistadas com o varejo será analisada em detalhe na próxima subseção.

Tem lojas que o seu 42 vai ser 46 , sabe? Eu costumo comprar em lojas que eu sei que tenha [...] Eu falei de fast fashion, eu gosto de comprar em lojas, sabe, que trocam de coleção, mais de uma, sabe? [...] Eu entrei na Luigi Bertolli, outro dia, no Ribeirão Shopping. Fui lá, nada me serve naquela loja, nada.

\section{Varejo: a epopeia emocional e o reforço do estigma}

Como em Sandicki e Ger (2010) ou Scaraboto e Fischer (2013), as consumidoras estudadas enfrentam dificuldades em consumir moda pela escassez de oferta. A escassez reforça o estigma, devido à constatação de que as peças nas lojas não são adequadas aos seus corpos. A percepção geral das entrevistadas é de que a numeração das lojas não é padronizada, ou seja, que os tamanhos com números iguais variam entre as diversas confecções. 
As tensões que surgem nos projetos identitários das consumidoras (justificativa, não aceitação e conformação) ligam-se à resposta que o varejo dá à sua aparência. 0 tamanho das roupas é uma fonte de angústia para as entrevistadas, como ilustrado pelo comentário da Marina:

Nunca tem G [...] e eu fico pensando [...] E daí eu vejo uma mulher, muito mais magra do que eu, levando G, aí eu falo: “É, tipo, será que tá tão apertado em mim? E eu não estou sentindo? Ou será que está tão largo nela?".

O estigma de estar acima do peso é reforçado pela escassez. Uma das alternativas que existem contra a escassez é recorrer a lojas especializadas no público plus size, contudo a percepção das consumidoras é de que esse mercado especializado não necessariamente contempla os objetos e marcas desejadas para completar seus projetos identitários.

As entrevistas indicam que as consumidoras cujo envolvimento com marcas conhecidas e tendências mais recentes é menor tendem a apreciar mais os produtos vendidos nesse tipo de loja do que aquelas cujo interesse por moda é mais relevante para a definição de suas identidades, corroborando, assim, a sugestão de Haytko (2004), para quem a experiência de varejo deriva, entre outros fatores, da consciência sobre as tendências.

Algumas das críticas às lojas plus size são retratadas nos comentários de Tarsila e Larissa, respectivamente:

Gente, cada coisa, aqueles panos que acho que esquentam mais que plástico! Não, e umas estampas nada a ver, uns negócios com uns fiozinhos dourados, sabe? Ai, muito assim... Ai, meio ultrapassado.

Hoje tem muita loja, assim, lojinha de bairro. Lá onde eu dou aula, por exemplo, tem muita loja que coloca os dois manequins para mostrar que têm uma padronagem maior. Então, nesses bairros de baixa renda, tem muita roupa do estilo periguete, né? Então, roupinha curtinha e uma cor muito forte ou muita estampa, esse tipo de coisa... E tem o manequim normal magrinho e tem o manequim maiorzinho. E é sempre uma roupa horrorosa, né? Assim, nesse manequim.

Na percepção das entrevistadas, as lojas plus size disponibilizam produtos cuja imagem não transmite jovialidade e sensualidade, salvo algumas raras exceções. Mesmo as con- sumidoras mais velhas buscam jovialidade e sensualidade, ainda que não desejem ser "periguetes", ou mulheres hipersensuais, com argumenta Tarsila. 0 fato de as roupas plus size ainda serem percebidas como roupas sombrias reforça a ideia de que a consumidora gorda é necessariamente feia e precisa se esconder.

Uma segunda saída para contornar a escassez é fazer compras em locais diferentes daqueles onde a consumidora mora. Esses locais podem ser cidades maiores, onde há maior variedade (geralmente São Paulo), ou cidades no exterior (geralmente Miami). Essa é uma forma de aumentar a escolha, no caso de viagens a cidades dentro do Brasil, e também de ter acesso a marcas famosas, com preços mais acessíveis e disponibilidade de peças na numeração adequada, quando se trata de compras no exterior. Como afirma Larissa:

Comprei na Zara, que eu nunca tinha comprado aqui no Brasil, lá fora eu falei: "Não é possível que eu estou comprando na Zara aqui fora e não compro no Brasil!" Mas é porque eu achava muita roupa 44 lá, eu olhava lá para a arara e você achava o seu número, aí você ia experimentar e o negócio entrava. Isso é muito chato [...] Você vai numa loja, você quer é comprar, você está com o dinheiro para comprar e não acha o negócio que você quer, aqui acontece muito isso. Lá aconteceu muito pouco.

Uma terceira saída para a escassez encontra-se na relação com as vendedoras. Muitas entrevistadas disseram frequentar lojas que vendem números como 50, 52 e 54, mas não divulgam essa informação ostensivamente. Tais lojas são, na descrição de Fabíola, butiques, lojas tradicionais, diferentes das lojas de departamentos. As informações são coproduzidas entre vendedoras e consumidoras (COVA e DALLI, 2002), em uma relação na qual a vendedora controla as informações, podendo passá-las ou não. Configura-se, assim, uma relação de poder entre as vendedoras e as consumidoras. Essas relações de poder envolvem a vendedora como parceira, como sacerdotisa ou como atormentadora (FISCHER e outros, 2009), esse último papel estigmatizante.

As vendedoras, como parceiras, adotam um comportamento solidário com as clientes e usam uma ampla gama de ferramentas de comunicação (telefone, e-mail ou celular), anunciando a chegada de peças de maneira antecipada. Essas vendedoras desenvolvem um relacionamento que beira a amizade com as suas clientes e tendem a "levá-las" com elas quando mudam de loja. 
Já as vendedoras que assumem o papel de sacerdotisas avisam quando os produtos estão disponíveis na loja, guardam as peças para as consumidoras e enviam sacolas com mercadorias a suas casas. Marlene chama esse mercado paralelo de “câmbio negro", e o caráter sacerdotal justifica-se pela distribuição de informação e recursos, que se encontra nas mãos das vendedoras, aumentando a escassez para as consumidoras que não fazem parte de suas "fiéis".

Por fim, existe o perfil das vendedoras como atormentadoras. Tais vendedoras utilizam a relação de poder de maneira a fidelizar a consumidora por meio ao acesso controlado das roupas plus size. A experiência de Marina traduz essa relação. Ela conta que, quando comprava em lojas nacionais (hoje prefere comprar no exterior), ficava amiga das vendedoras e, com frequência, encomendava as peças $\mathrm{G}$ no catálogo. Mas ela diz: "Ela (a vendedora) não vai te mostrar o catálogo se você não for lá pelo menos uma vez por semana comprar uma coisinha" e emenda: "Eu tinha amizade (com vendedoras) em todas as lojas".

\section{DISCUSSÃO}

Exploramos como o estigma e a escassez no varejo afetam os projetos de identidade de consumidoras plus size e como essas consumidoras contornam a escassez para tornar reais seus eu -estendidos desejados.

As entrevistas indicam que a moda é um elemento significativo à definição identitária das consumidoras, o que está em consonância com a literatura anterior explorada pelo tema (MURRAY, 2002; THOMPSON e HAYTKO, 1997). Mas o estigma que rodeia as mulheres plus size, reforçado pelo varejo de moda, leva-as a ligarem suas identidades de moda ao seu peso, de duas formas distintas: (1) aceitando-o e buscando alternativas no varejo para sentirem-se incluídas na moda (SCARABOTO e FISCHER, 2013) e (2) rejeitando-o e buscando conformar-se com normas de beleza padrão, enquanto se justificam moralmente pela sua condição (THOMPSON e HIRSCHMAN, 1995). As duas situações não são excludentes e estão presentes muitas vezes nas narrativas de uma mesma entrevistada, fazendo parte de uma identidade diversificada, que vai ganhando substância e reflexão ao longo dos anos.

Tais descobertas estão em consonância com trabalhos que exploram a questão da identidade na contemporaneidade. A busca por um "eu" coerente (SHANKAR, ELLIOT, FITCHETT, 2009) perpassa a fragmentação pós-moderna (FIRAT e VENKATESH, 1995). Contudo, a visão identitária positiva exposta por Firat e Venkatesh (1995) não considera o corpo socializado
(THOMPSON e HIRSCHMAN, 1995), que depende da alteridade (OLIVEIRA e LEÃO, 2012) para ser aceito e legitimado (SANDICKI e GER, 2010).

O varejo entra como problema e solução para as mulheres plus size. Ao mesmo tempo que reforça a estigmatização por meio de discursos transmitidos (DION e ARNOULD, 2011) e promove a exclusão dos estigmatizados (GOFFMAN, 1963), também traz possíveis soluções, restringindo ou possibilitando a materialização dos objetos que constituem o eu-estendido, ou seja, os discursos do varejo são ambíguos e interpretados pelas consumidoras de maneiras distintas (THOMPSON, RINDFLEISCH, ARSEL, 2006), contudo quase sempre pela ótica do corpo socializado (THOMPSON e HIRSCHMAN, 1995). Elas encontram o acolhimento de algumas vendedoras e as soluções específicas de algumas lojas ou marcas, mas se sentem excluídas em determinados ambientes. Tanto o acolhimento quanto a exclusão ocorrem por causa do formato dos seus corpos.

Quando estigmatizadas ou excluídas, recorrendo majoritariamente a lojas plus size cujas ofertas, muitas vezes, não contemplam marcas ou produtos percebidos como sofisticados, as consumidoras que buscam um eu-estendido (BELK, 1988) mais tradicionalmente fashion não realizam completamente seus projetos identitários. Quando contempladas pelo varejo tradicional em termos de oferta de roupas, as consumidoras buscam a parceria das vendedoras para ter acesso a tais objetos, que, mesmo quando disponíveis, são bastante disputados.

0 "câmbio negro" com as vendedoras mostra mais um aspecto de poder na relação entre as consumidoras e o varejo. A experiência de consumo (MEYER e SCHWAGER, 2007) passa a ser (des) legitimadora da identidade da consumidora que busca superar o corpo socializado, enquanto molda sua identidade de moda nele, sendo acolhedora ou perturbadora, a depender dos agentes envolvidos nessa experiência (em especial, as vendedoras).

Há, também, um sinal de que as consumidoras plus size estejam engajando-se em soluções criativas para serem incluídas no mundo da moda (SCARABOTO e FISCHER, 2013), como o acesso a blogs de moda plus size e compras em locais distintos de suas cidades. Novas lojas plus size e marcas que vêm surgindo no varejo brasileiro podem vir a oferecer novas narrativas identitárias, cujos significados sejam cocriados pelos consumidores (VARGO e LUSCH, 2004), especialmente na internet, uma alternativa para a compra que tem ganhado importância. Esse formato de loja pode permitir acesso aos produtos, a despeito de a compra não permitir a experimentação e gerar muito retorno de produto (THE ECONOMIST, 2013a). Este é um aspecto que pode ser explorado em pesquisas futuras. 


\section{IMPLICAÇÕES GERENCIAIS}

Dados estatísticos oficiais indicam que $48,5 \%$ da população brasileira estão com peso considerado superior ao ideal (BRASIL, 2012), tendência similar à de outros países da América Latina, nos quais comidas do tipo fast-food popularizaram-se nas últimas décadas (THE ECONOMIST, 2013b). Essas pessoas representam um novo e expansivo mercado para o varejo de moda no Brasil. Contrariando resultados de trabalhos anteriores (CHATTARAMAN e RUDD, 2006), mas assemelhando-se a resultados de outros (OTIENO, HARROW, LEAGREENWOOD, 2005), nossa pesquisa mostrou que o interesse da mulher plus size em moda existe e se manifesta na maneira criativa pela qual ela compra as peças que utiliza. Marcas que busquem atender o público plus size podem incorporar cores, criatividade e até assinaturas de estilistas em suas peças, já que, por mais que as consumidoras lutem com o peso e tenham sua identidade marcada pela condição de plus size, há um movimento de valorização da autoestima dessas mulheres.

Como visto nas análises dos dados, o varejo pode tornarse um instrumento de estigmatização para essas mulheres, de modo que a experiência de compra, que poderia ser lúdica e divertida, torna-se insatisfatória e (até) opressiva. Vários elementos colaboram com tal situação: a falta de padronização da numeração das roupas, a disponibilidade das peças, a sua qualidade e o tratamento dado pelas vendedoras. Seja o varejis ta voltado ou não exclusivamente para esse público, medidas como minimizar a variação da numeração das peças em uma mesma loja ou marca, disponibilizar peças que estejam nas tendências atuais, manter as vendedoras treinadas para lidar com esse público em particular e evitar práticas discriminatórias podem tornar a experiência de compra mais agradável e aumentar o ticket médio dessa cliente.

Cabe a cada varejista ou marca decidir se esse será um público a ser atendido ou não. Caso a decisão seja positiva, a representação de mulheres plus size na comunicação da marca deve ser discutida. Modelos plus size não necessariamente ostentam manequins acima do 44 (existem aquelas que usam manequim 42 ou até 40) e, visualmente, dificilmente seriam percebidas como gordas. Se um varejista busca exclusivamente o público plus size, talvez a escolha de modelos que realmente pareçam estar acima do peso seja mais bem-sucedida.

O segmento on-line parece ser uma forma de investimento promissora para o público em questão, especialmente quando o varejista volta-se exclusivamente à consumidora plus size. Mesmo com algumas desvantagens que tal modali- dade traz para a consumidora, como a impossibilidade de se experimentarem as roupas, empresas diversas vêm trazendo inovações para melhorar a experiência de compra on-line ou integrar as lojas on-line às lojas convencionais de varejo (THE ECONOMIST, 2013a). Os investimentos em tal modalidade parecem ser uma oportunidade para o varejista, especialmente aquele que busca trabalhar com um público exclusivamente plus size, atendendo às necessidades fashion dessas consumidoras, que querem ser parte do mundo da moda e estão dispostas a gastar com isso.

\section{LIMITAÇÕES E SUGESTÕES DE PESQUISAS FUTURAS}

0 artigo apresenta algumas limitações. Primeiramente, as diferenças demográficas e geográficas poderiam estar mais acentuadas. Mesmo tendo entrevistado pessoas com perfis socioeconômicos distintos, pela percepção dos autores, nenhuma das consumidoras enquadra-se em um perfil de classe $D$ ou mais baixa. No mais, apenas duas das entrevistadas são negras, o que pode ter trazido um viés aos dados, considerando que há indícios de que a influência dos padrões de beleza seja menor em grupos de pessoas negras (EVANS e MCCONNELL, 2003).

Se a identidade na moda passa por uma questão do corpo socializado (THOMPSON e HIRSCHMAN, 1995), há também indícios de que ela seja influenciada por questões de classe e renda, estruturais, e que impedem o consumidor de constituir sua identidade criativamente (MURRAY, 2002).

Perguntas também persistem em relação às estratégias varejistas. 0 mercado de moda plus size parece crescer e ganhar legitimidade entre algumas das entrevistadas, que já se associam a uma identidade plus size. Tal aspecto poderia ser trabalhado à luz da ideologia de varejo (BORGHINI e outros, 2009), buscando entender como acontece a apropriação e a interpretação dessa ideologia pelas consumidoras, especialmente em um estudo in loco.

Por fim, estando tanto questões identitárias quanto discursos transmitidos por objetos e lugares ligados a marcas (FOURNIER, 1998; THOMPSON, RINDFLEISCH, ARSEL, 2006), existe uma oportunidade de aprofundar o estudo do consumo e do varejo plus size em relação a elas, por meio de questões como: (1) A escassez de marcas consagradas afeta a identidade das consumidoras? (2) Qual a perspectiva dos varejistas dessas marcas e suas razões para não estarem no mundo plus size? (3) Existe espaço para uma marca exclusivamente plus size atingir um potencial simbólico de luxo? 


\section{REFERÊNCIAS}

AHUVIA, A. C. Beyond the extended self: loved objects and consumers' identity narratives. Journal of Consumer Research, v. 32, n. 1, p. 171-184, 2005.

ALBINO, J. C. A; RESENDE, C. A; SIQUEIRA, H. J. H; CARRIERI, A. P. Cultura de consumo, comunicação e práticas de branding: aproximações possíveis. In: Encontro de Marketing do ANPAD, 4, 2010, Florianópolis. Anais. Florianópolis: ANPAD, 2010.

ALMEIDA, M. I. M. Nada além da epiderme: a performance romântica da tatuagem. In: BARBOSA, L; CAMPBELL, C. (Org) Cultura, consumo e identidade. Rio de Janeiro: FGV, 2006. p. 107-136.

ARNOLD, S. J; FISCHER, E. Hermeneutics and consumer research. Journal of Consumer Research, v. 21, n. 1, p. 55-70, 1994.

ARNOLD, S. J; KOZINETS, R. V; HANDELMAN, J. M. Hometown ideology and retailer legitimation: the institutional semiotics of Wal-Mart flyers. Journal of Retailing, v. 77, n. 2, p. 243-271, 2001.

ARNOULD, E; THOMPSON, C. J. Consumer culture theory (CCT): twenty years of research. Journal of Consumer Research. v. 31, n. 4, p. 868-882, 2005.

ARSEL, Z; THOMPSON, C. J. Demythologizing consumption practices: how consumers protect their field-dependent identity investments from devaluing marketplace myths. Journal of Consumer Research, v. 37, n. 5 , p. 791-806, 2011.

ASKEGAARD, S; LINNET, J. T. Towards an epistemology of consumer culture theory: phenomenology and the context of context. Marketing Theory, v. 11, n. 4, p. 381-404, 2011.

BAHL, S. The dialogical consumer self. In: RUVIO, A. A; BELK, R. W. (Ed) The Routledge companion to identity and consumption. New York: Routledge, 2013.

BELK, R. W. Possessions and the extended self. The Journal of Consumer Research, v. 15, n. 2, p. 139-168, 1988.

BLEICHER, J. Hermenêutica contemporânea. Rio de Janeiro: Edições 70, 1980. 383 p.

BORGHINI, S. e outros. Why are themed brandstores so powerful? Retail brand ideology at American Girl Place. Journal of Retailing, v. 85, n. 3, p. 363-375, 2009.

BRASIL, Portal da saúde do SUS, 2011. Disponível em: http:// portalsaude.saude.gov.br/portalsaude/noticia/4718/162/quasemetade-da-populacao-brasileira-esta-acima-do-peso.html. Acesso em 06.06.2012.

CHATTARAMAN, V; RUDD, N. Preferences for aesthetic attributes in clothing as a function of body image, body cathexis and body size. Clothing and Textiles Research Journal, v. 24, n. 1, p. 46-61, 2006.

COVA, B; DALLI, D. Working consumers: the next step in marketing theory? Marketing Theory, v. 9, n. 3, p. 315-339, 2009.

DION, D; ARNOULD, E. Retail luxury strategy: assembling charisma through art and magic. Journal of Retailing, v. 87, n. 4, p. 502-520, 2011.

DOUGLAS, M; ISHERWOOD, B. The world of goods: towards an anthropology of consumption. New York: Routledge, 1979. $200 \mathrm{p}$.

EVANS, P. C; McCONNELL, R. A. Do racial minorities respond in the same way to mainstream beauty standards? Social comparison processes in Asian, black, and white women. Self and Identity. v. 2, n. 2 p. 153-167, 2003.
FIRAT, A. F; VENKATESH, A. Liberatory postmodernism and the reenchantment of consumption. Journal of Consumer Research, v. 22, n. 3, p. 239-267, 1995.

FISCHER, E. e outros. Are we there yet? Co-producing succeed and failure in a "consumer-intensive" service context. In: SHERRY, J; FISCHER, E. Exploration in consumer culture theory. Routledge: New York, 2009.

FONTENELLE, I. A. Corpo e marca publicitária na sociedade das imagens. Comunicação, Mídia e Consumo, v. 1, n. 1, p. 05-16, 2004.

FOURNIER, S. Consumers and their brands: developing relationship theory in consumer research. Journal of Consumer Research, v. 24, n. 4, p. 343-353, 1998.

GAIÃO, B. F; SOUZA, L. L; LEÃO, A. S. Consumer culture theory (CCT) já é uma escola de pensamento em marketing? RAE-Revista de Administração de Empresas, v. 52, n. 3, p. 330-344, 2012.

GAO, L. S; WHEELER, C; SHIV, B. The "shaken self": product choices as a means of restoring self view confidence. Journal of Consumer Research, v. 36, n. 1 , p. 29-38, 2009.

GOFFMAN, E. Stigma: notes on the management of spoiled identity. [S.1]: Touchstone, 1963. $124 \mathrm{p}$.

HAYTKO, D. L; BAKER, J. It's all at the mall: exploring adolescent girls' experiences. Journal of Retailing, v. 80, n. 1, p. 67-83, 2004.

HOLT, D. Why do brands cause trouble? A dialectical theory of consumer culture and branding. Journal of Consumer Research, v. 29, n. 1, p. 7090, 2002.

HOPKINSON, G.C.; HOGG, M.K. Stories: how they are used and produced in market(ing) research. In: BELK, Russell W. (Ed.). Handbook of qualitative research methods in marketing. Edward Elgar Publishing: Northampton, MA, USA, 2006.p. 156-174.

KATES, S. M. The protean quality of subcultural consumption: an ethnographic account of gay consumers. Journal of Consumer Research, v. 29, n. 3, p. 383-399, 2002.

KLEINE III, R. E; KLEINE, S. S; KERNAN, J. B. Mundane consumption and the self: a social-identity perspective. Journal of Consumer Psychology, v. 2, n. 3, p. 209-235, 1993.

KOZINETS, R. V. Can consumers escape the market? Emancipatory illuminations from burning man. Journal of Consumer Research, v. 29, n. 1, p. 20-38, 2002.

KOZINETS, R. V. Utopian enterprise: articulating the meanings of star trek's culture of consumption. Journal of Consumer Research, v. 28, n. 1, p. 67-88, 2001.

LUEDICKE, M. K; THOMPSON, C. J; GIESLER, M. Consumer identity work as moral protagonism: how myth and ideology animate a brandmediated moral conflict. Journal of Consumer Research, v. 36, n. 6, p. 1016-1032, 2010.

MEYER, C; SCHWAGER, A. Understanding customer experience. Harvard Business Review, n. 26, v. 117, p. 1-11, 2007.

MOISANDER, J; VALTONEN, A. Qualitative marketing research: a cultural approach. Thousand Oaks: SAGE, 2006.

MURRAY, J. B. The politics of consumption: a re-inquiry on Thompson and Haytko's (1997) “Speaking of Fashion”. Journal of Consumer Research, v. 29, n. 3, p. 427-440, 2002.

OLIVEIRA, M. C; LEÃO, A. S. Sendo aos olhos do outro: o papel da alteridade na construção da identidade metrossexual. RAUSP-Revista de Administração da Universidade de São Paulo, v. 47, n. 2, p. 264-274, 2012. 
OTIENO, R; HARROW, C; LEA-GREENWOOD, G. The unhappy shopper, a retail experience: exploring fashion, fit and affordability. International Journal of Retail \& Distribution Management, v. 33, n. 4, p. 298-309, 2005.

SANTOS, L. S; PINTO, M. R. Em busca de uma trilha interpretativista para a pesquisa do consumidor: uma proposta baseada na fenomenologia, na etnografia e na grounded theory. RAE-Revista de Administração de Empresas, v. 7, n. 2, p. 1-28, 2008.

SAMPAIO, C. H. e outros. Pesquisa científica da área de marketing no Brasil: uma revisão da primeira década do século 21. RAC-Revista de Administração Contemporânea, v. 16, n. 3, p. 459-478, 2012.

SANDICKI, Ö; GER, G. Veiling in style: how does a stigmatized practice become fashionable? Journal of Consumer Research, v. 37, n. 1, p. 15-36, 2010.

SANDICKI, Ö; GER, G. Stigma, identity and consumption. In: RUVIO, A. A; BELK, R. W. (Ed) The Routledge companion to identity and consumption. New York: Routledge, 2013.

SCARABOTO, D; FISCHER, E. Frustrated fatshionistas: an institutional theory perspective on consumer quests for greater choice in mainstream markets. Journal of Consumer Research, v. 39, n. 6, p. 1234-1257, 2013.

SCHAU, H. J; GILLY, M. C. We are what we post? Self-presentation in personal web space. Journal of Consumer Research, v. 30, n. 3, p. 385 404, 2003

SCHAU, H. J; GILLY, M. C; WOLFINBARGER, M. Consumer identity renaissance: the resurgence of identity inspired consumption in retirement. Journal of Consumer Research, v. 36, n. 2, p. 255-276, 2009.

SHANKAR, A; ELLIOTT, R; FITCHETT, J. A. Identity, consumption and narratives of socialization. Marketing Theory, v. 9, n. 1, p. 75-94, 2009.

SCHOUTEN, J. W.; MCALEXANDER, J. H. Subcultures of consumption: an ethnography of the new bikers. Journal of Consumer Research, v. 22, n. 1, p. 43-61, 1995.
THE ECONOMIST. Obesity in Latin America: battle of the bulge. 27.07.2013b. Disponivel em: http://www.economist.com/news/ americas/21582273-waistlines-grow-so-does-fight-against-junk-foodbattle-bulge. Acesso em: 27.07.2013.

THE ECONOMIST. Shopping: the emporium strikes back. 13.07.2013a Disponivel em: http://www.economist.com/news/briefing/21581755. retailers-rich-world-are-suffering-people-buy-more-things-online-theyare-finding. Acesso em: 13.07.2013.

THOMPSON, C. J. Interpreting consumers : a hermeneutical framework for deriving marketing insights from the texts of consumers' consumption stories. Journal of Marketing Research, v. 34, n. 4, p. 438-455, 1997.

THOMPSON, C; HAYTKO, D. Speaking of fashion: consumers' uses of fashion discourses and the appropriation of countervailing cultural meanings. Journal of Consumer Research, v. 24, n. 1, p. 15-42, 1997.

THOMPSON, C. J; HIRSCHMAN, E. C. Understanding the socialized body: a poststructuralist analysis of consumers' self-conceptions, body images, and self-care practices. Journal of Consumer Research, v. 22, n. 2, p. 139-153, 1995.

THOMPSON, C. J; LOCANDER, W; POLLIO, R. Putting consumer experience back into consumer research: the philosophy and method of existentialphenomenology. Journal of Consumer Research, v. 16, n. 2, p. 133-146, 1989.

THOMPSON, C. J; TROESTER, M. Consumer value systems in the age of postmodern fragmentation: the case of the natural health microculture. Journal of Consumer Research, v. 28, n. 4, p. 550-571, 2002.

THOMPSON, C. J; RINDFLEISCH, A; ARSEL, Z. Emotional branding and the strategic value of the Doppelgänger brand image. Journal of Marketing, v. 70, n. 1, p. 50-64, 2006.

WINN, J. Making it big. Entrepreneurship theory and practice, v. 28, n. 5 , p. 487-500, 2004.

VARGO, S. L; LUSCH, R. F. Evolving to a new dominant logic for marketing. Journal of Marketing, v. 68, n. 1, p. 1-17, 2004. 